\title{
Aspectos Cognitivos e Comportamentais na Média Meninice de Crianças Nascidas Pré-Termo e com Muito Baixo Peso ${ }^{1}$
}

\author{
Maria Beatriz Machado Bordin², \\ Maria Beatriz Martins Linhares e Salim Moysés Jorge \\ Universidade de São Paulo, Ribeirão Preto
}

\begin{abstract}
RESUMO - O objetivo deste estudo consiste em avaliar o desenvolvimento cognitivo e o comportamento de 20 crianças de 8 a 10 anos nascidas pré-termo e baixo peso $(\leq 1500 \mathrm{~g})$ no HCFMRP. Na avaliação cognitiva utilizou-se o RAVEN, o WISC e a avaliação assistida e na avaliação do comportamento utilizou-se a Escala de Rutter. Verificou-se que as crianças apresentaram desempenho intelectual na média, tendendo ao rebaixamento cognitivo. Na avaliação cognitiva assistida, a maior parte das crianças foi classificada como "ganhadora" e foram capazes, com a ajuda da examinadora, de implementar e manter estratégias eficientes para a resolução do problema. Quanto ao comportamento, foram detectados índices sugestivos de necessidade de atendimento psicológico para a maioria das crianças. Verificaram-se correlações significativas entre os resultados das avaliações cognitivas entre si e entre estas e a do comportamento. Os resultados apontam para a necessidade de seguimento psicológico longitudinal desse grupo de risco para problemas de desenvolvimento.
\end{abstract}

Palavras-chave: pré-termo; muito baixo peso; desenvolvimento cognitivo.

\section{Cognitive and Behavioral Aspects of Preterm Low Birthweight Children at School Age}

\begin{abstract}
The aim of this study was to assess cognitive development and behavior of 20 preterm and very low birthweight children at school age. For the cognitive assessment it was used psychometric assessment (RAVEN and WISC) and assisted assessement approaches. Rutter's Scale was used to assess behavior. Results show that children presented average intellectual level in the psychometric tests used, with a tendency of cognitive impairment. The results of the assisted cognitive assessment showed that most children were classified as "gainners"; the children improved their performance with the examiner's assistance and were able to implement efficient strategies to solve the task problem. Behavioral data indicated the necessity of psychological treatment for most children. There were correlations between the results of the cognitive assessments themselves and between results of the cognitive assessment and the behavioral assessment. These results indicate the need to follow-up the high risk neonatal child's psychological development.
\end{abstract}

Key words: pre-term; very low birth weight; cognitive development.

Nos últimos anos tem crescido o interesse de profissionais e pesquisadores que atuam na área da Saúde em favorecer as condições de vida da criança, procurando garantir sua boa adaptação no decorrer de seu desenvolvimento. Reflete-se, desta forma, em um investimento cada vez maior em estudos que tenham como meta a prevenção de problemas, por meio da identificação de fatores de risco ao desenvolvimento infantil.

Lewis, Dlugokinski, Caputo e Griffin (1988) destacam três conjuntos de fatores que devem ser considerados na identificação de crianças com risco de problemas de desenvolvimento: a) fatores de risco da própria criança (componentes biológicos, temperamento e sintomatologia); b) fatores de risco do ambiente familiar (história parental e funcionamento familiar) e da comunidade (suporte social, escola, contexto

1 Este trabalho faz parte da Dissertação de Mestrado da primeira autora sob orientação da segunda, a qual foi realizada com bolsa da FAPESP.

2 Endereço: Av. Nove de Julho 980/Depto. Neurologia, Psiquiatria e Psicologia Médica, CEP: 14025-000/Ribeirão Preto-SP.

E-mail: beatriz.bordin@netsite.com.br cultural, nível sócio econômico). No primeiro conjunto incluem-se as condições de prematuridade e baixo peso ao nascimento, que são fatores orgânicos de alto risco ao desenvolvimento infantil.

O Centro Nacional de Epidemiologia, órgão vinculado ao Ministério da Saúde, realizou recentemente um levantamento para configurar "o mapa dos nascidos vivos" no Brasil. De acordo com esse estudo, 212 mil bebês nasceram com baixo peso no país em 1996, o que corresponde a $8 \%$ do total de crianças nascidas nesse mesmo período. Além disso, 18.100 crianças nasceram até o sétimo mês, ou seja, nasceram pré- termo. Os dados revelam ainda que a taxa de mortalidade é maior no caso de crianças nascidas prematuras e/ou com baixo peso (Bernardes, 1998).

Devido aos avanços na área de Neonatologia, tem havido um aumento na taxa de sobrevivência de recém-nascidos pré-termo com muito baixo peso, desde que recebam atendimento especializado em UTI neonatal. Diante disso, Beckwith e Rodning (1991) propõem uma subdivisão na classificação de crianças nascidas com peso $\leq 2.500 \mathrm{~g}$. São consideradas crianças muito baixo peso aquelas nascidas com peso $<1.500 \mathrm{~g}$, enquanto que as crianças com peso ao nasci- 
mento $<1.000 \mathrm{~g}$ são definidas como de extremamente baixo peso. Particularmente esses dois subgrupos constituem-se em crianças vulneráveis com risco de problemas de crescimento físico e de desenvolvimento psicológico.

De um lado, no campo da Medicina tem havido contribuições inestimáveis no que se refere ao aperfeiçoamento da assistência médica, produzindo conseqüentemente aumento da sobrevida dessas crianças; por outro lado são poucos os estudos em nosso meio que esclarecem sobre o desenvolvimento e as condições de vida dessas crianças do ponto de vista psicológico. Novello, Degraw e Kleinman (1992) salientam a importância de se acompanhar o desenvolvimento de crianças com problemas neonatais, a fim de evitar que se potencializem os fatores de risco, transformando-se em problemas.

Crianças nascidas pré-termo com baixo peso quando comparadas a crianças nascidas com peso $\geq 2.500 \mathrm{~g}$ ou a termo são mais propensas a apresentar dificuldades cognitivas (Bradley \& cols.,1993; Dammann \& cols., 1996; Smedler, Faxelius, Bremme \& Lagerström,1992; Waber \& McCornick, 1995; ), de desempenho escolar (Taylor, Hack, Klein \& Schatschneider, 1995), comportamentais (Brandt, Magyary, Hammond \& Barnard, 1992) e de crescimento físico (Werner,1986). Ressalta-se porém, que as condições neurológicas (Antunha, 1994; Brazelton, 1994; Fitzhardinze \& Steven, 1972) e familiares (Bradley \& cols., 1993; Bradley \& cols., 1994; Liaw \& Brooks-Gunn, 1993; Werner,1986) da criança atuam de modo significativo a atenuar ou agravar o desencadeamento dessas dificuldades.

A busca de entendimento acerca do desenvolvimento cognitivo de crianças com baixo peso ao nascer tem motivado várias pesquisas que avaliam o nível intelectual dessas crianças em diferentes idades, visto que a cognição constituise em variável importante na estruturação e dinâmica do desenvolvimento global da criança e pode fornecer indicadores sugestivos acerca do seu desempenho futuro. Sansavini, Rizzardi, Alessandroni e Giovanelli (1996) relataram que crianças nascidas pré-termo com muito baixo peso apresentaram desenvolvimento cognitivo mais rebaixado quando comparadas a crianças controle. As primeiras mostraram atrasos cognitivos, motores e sociais no primeiro ano de vida, atrasos linguíísticos até 24 meses e atrasos cognitivos gerais até cinco anos, em comparação com as segundas. Contudo, os escores das crianças pré- termo nos testes encontravamse dentro da faixa de normalidade.

Dammann e cols. (1996) avaliaram crianças nascidas com muito baixo peso $(\leq 1.500 \mathrm{~g})$ com o objetivo de examinar seu desempenho cognitivo através de avaliação neuropediátrica e psicológica. Foram diagnosticadas desordens neurológicas aos seis anos de idade em $64 \%$ das crianças estudadas. De um lado, a morbidade neurológica influenciou o desenvolvimento cognitivo em todos os domínios avaliados. Por outro, o nível social das crianças influenciou o seu desempenho, principalmente nos domínios da inteligência e linguagem. Além disso, crianças nascidas com muito baixo peso com hiperatividade, desajeitadas ("clumsy") ou com paralisia cerebral diferiram significativamente daque- las sem prejuízo neurológico nos domínios da inteligência, habilidades viso- motoras e memória visual.

A maioria dos estudos que vêm demonstrando que crianças nascidas com baixo peso apresentam risco na sua evolução, deixam claro também que, quanto mais baixo o peso mais baixo o coeficiente de inteligência e, conseqüentemente, maior é a probabilidade de desenvolverem dificuldades de aprendizagem. Waber e McCornick (1995) examinaram 635 crianças, subdivididas em quatro grupos: extremamente muito baixo peso $(\leq 1.000 \mathrm{~g})$, muito baixo peso $(1.001 \mathrm{~g}$ $1500 \mathrm{~g})$, baixo peso $(1.501 \mathrm{~g}-2500 \mathrm{~g})$ e com peso adequado para a idade gestacional $(>2.500 \mathrm{~g})$. Os resultados apontaram a existência de deficiência cognitiva, principalmente nos grupos extremamente baixo peso e muito baixo peso. As crianças extremamente baixo peso também apresentaram dificuldades em matemática e no processamento visual em maior proporção do que as demais. Taylor e cols. (1995) demonstraram que crianças com menos de $750 \mathrm{~g}$ e sem problemas neurológicos, obtiveram escores mais baixos nos testes de desempenho matemático e na área de reconhecimento de palavras quando comparadas a crianças com peso entre $750 \mathrm{~g}$ e $1.499 \mathrm{~g}$.

Apesar de o baixo peso ser considerado uma condição adversa significativa para o desenvolvimento da criança, não significa que este fator, por si só, implique em comprometimento do desenvolvimento psicológico, como demonstram os estudos sobre resiliência, definida como a capacidade de enfrentar efetivamente o estresse interno e externo, apesar das adversidades (Werner, 1986). Bradley e cols. (1994) avaliaram 243 crianças prematuras (idade gestacional $\leq 37$ semanas) e de baixo peso $(\leq 2.500 \mathrm{~g})$ vivendo em condições de pobreza, a fim de verificar se a qualidade dos cuidados que tais crianças recebiam proporcionavam a elas alguma medida de proteção contra as conseqüências da pobreza e da prematuridade. Somente 26 foram identificadas com funcionamento normal quanto aos aspectos cognitivo, social/ adaptativo, de saúde e de crescimento. Estas crianças, que mostraram sinais de resiliência, diferiram de crianças não resilientes no que se refere ao ambiente familiar: recebiam mais aceitação, estimulação e cuidados, e também viviam em condições de segurança e em lares com menos pessoas. Havia, também, indicações de que a inteligência materna e a saúde perinatal adequada da criança foram fatores que forneceram alguma medida de proteção para as crianças prematuras que viviam em condições de pobreza.

Parece que paralelamente às dificuldades no âmbito cognitivo de crianças que nascem em condições pré-termo com baixo peso, encontram-se estudos que mostram que elas podem se desenvolver adequadamente, desde que devidamente estimuladas pelo ambiente. Kalmár (1996) ressalta que a qualidade do ambiente familiar tem mais peso do que fatores de risco perinatais em sua capacidade para predizer resultados a longo prazo. Mutch, Leyland e McGee (1993) e Forslund e Bjerre (1990) destacam a classe social e o nível educacional da mãe como fatores relevantes para o desenvolvimento cognitivo na idade de quatro anos das crianças pré-termo. Brazelton (1994) mostra que, apesar de as crian- 
ças prematuras apresentarem dificuldades, tais como prejuízo neurológico, dificuldades de aprendizagem, distúrbios de atenção ou hiperatividade, essas podem apresentar recursos para recuperação do sistema nervoso, desde que um trabalho de intervenção precoce seja realizado por profissionais e familiares. Essa recuperação parece estar associada à plasticidade cerebral (Antunha, 1994).

$\mathrm{Na}$ avaliação das crianças pré-termo com baixo peso, além do desenvolvimento cognitivo, outra área investigada relaciona-se ao padrão comportamental alterado (Carvalho, Linhares \& Martinez, no prelo). Desde o nascimento essas crianças são percebidas por suas mães como mais irritáveis, choronas e hipoativas que as crianças nascidas a termo (LevyShiff, Einat, Mogilner, Lerman \& Krikler, 1994; Stjernqvist, 1993). Distúrbios de comportamento, tais como hiperatividade, problema de temperamento, desatenção, comportamento opositor, ansiedade, inibição social, tiques e comportamento estereotipado, são também observados no decorrer do desenvolvimento de crianças nascidas pré-termo com baixo peso em comparação com crianças a termo. Tais dificuldades são intensificadas quando a criança pré-termo apresenta complicações neonatais, como por exemplo, hemorragia intraventricular, prejuízo neurológico/sensorial, má formação genética e doenças metabólicas (Landry, Chapieski, Richardson, Palmer \& Hall, 1990; Laucht, Esser \& Schmit, 1997).

Weiglas-Kuperus, Koot, Baerts, Fetter e Sauer (1993) salientam que dois padrões de comportamento aparecem em maior escala em crianças nascidas pré- termo com baixo peso: depressivo (provavelmente relacionado à qualidade do relacionamento familiar) e de desatenção (provavelmente relacionado a anomalias cerebrais).

$\mathrm{Na}$ adolescência, crianças nascidas prematuras e com baixo peso tendem a apresentar desajustamento emocional, sinais de depressão e de ansiedade, agressividade e autoestima baixa no contexto familiar e escolar quando comparadas a grupos controle (Levy-Shiff, Einat, Har-Even, Mogilner, Lerman \& Krikler, 1994; Levy-Shiff, Einat, Mogilner \& cols., 1994).

A associação entre o fator de risco biológico da prematuridade e do baixo peso e fatores proximais de risco ambiental (alto nível de estresse familiar, baixa responsividade materna e dificuldade em exercer a maternidade) e/ou distais (baixo nível educacional e sócio-econômico dos pais, situação conjugal irregular) parecem contribuir para o surgimento de problemas de comportamento (Brandt \& cols.,1992; Gennaro, Brooten, Roncoli \& Kumar, 1993; Levy-Shiff, Einat, Mogilner \& cols., 1994; Sobotková, Dittrichová \& Mandys, 1996). Os fatores proximais atuam diretamente e no contexto atual de desenvolvimento da criança, enquanto que os fatores distais atuam indiretamente ou são condições antecedentes.

Considerando-se: a) a necessidade de avaliar crianças de risco nascidas pré-termo com muito baixo peso, a médio prazo, após a passagem do enfrentamento de tarefas evolutivas significativas de aquisição e domínio da linguagem e entrada na escola; b) a escassez de estudos de avalia- ção do desenvolvimento e aprendizagem dessas crianças em nosso meio, propõe-se o presente estudo. Este tem por objetivo $^{3}$ : a) avaliar aspectos do desempenho cognitivo e do comportamento de crianças na fase escolar, com idade de oito a dez anos, nascidas pré-termo com muito baixo peso ( $£$ $1.500 \mathrm{~g}$ ); b) proceder à avaliação cognitiva dessas crianças combinando duas abordagens: avaliação psicométrica da inteligência e avaliação assistida em situação de resolução de problemas (Linhares, 1996; Tzuriel \& Klein, 1987 ). Esta última modalidade de avaliação tem demonstrado ser útil com crianças com desvantagens, diagnóstico de deficiência mental ou com problemas de aprendizagem, por permitir diferenciações intra-grupo, revelando recursos potenciais individuais do ponto de vista cognitivo (Brown \& Ferrara, 1985; Linhares, 1996; Linhares, Santa Maria Escolano \& Gera, 1998; Santa Maria \& Linhares, 1999). Permite comparar o desempenho da criança em diferentes momentos de uma situação de resolução de problemas: sem ajuda do examinador, com assistência e após a suspensão da mesma, comparando o sujeito consigo mesmo no processo de avaliação.

\section{Método}

\section{Participantes}

A amostra foi composta por 20 crianças nascidas prétermo com peso abaixo de $1500 \mathrm{~g}$ no Hospital das Clínicas da Faculdade de Medicina de Ribeirão Preto da Universidade de São Paulo (HCFMRP) entre os anos de 1988 a 1991. Os sujeitos tinham de oito anos a dez anos e dois meses (Md $=9$ anos) e a maioria era do sexo feminino $(n=13)$. O peso ao nascimento dessas crianças variou de 540 a $1475 \mathrm{~g}(M d=$ $1210 \mathrm{~g}$ ). Oito das crianças avaliadas estavam cursando a $3^{\mathrm{a}}$ série, seis estavam cursando a $1^{\mathrm{a}}$ série e quatro a $2^{\mathrm{a}}$ série. Uma criança freqüentava escola especial, uma segunda criança freqüentava o pré-primário de uma sala especial para deficientes auditivos e uma terceira criança estava em classe especial de $1^{a}$ série para deficiente auditivo. Duas crianças apresentavam história de repetência escolar. A grande maioria $(75 \%)$ freqüentava série compatível com a idade cronológica, predominantemente em escola pública.

As mães das crianças eram jovens $(M d=33$ anos; $A V=$ 26-48 ) e o grau de escolaridade variava da $2^{\mathrm{a}}$ série incompleta do $1^{\circ}$ grau ao nível superior incompleto. Pouco mais da metade das mães das crianças avaliadas trabalhavam fora, exercendo atividades não qualificadas ou de qualificação inferior, segundo classificação de Soares e Fernandes (1989). Os pais, por sua vez, apresentavam grau de escolaridade variando da $2^{\mathrm{a}}$ série do $1^{\circ}$ grau ao $2^{\circ}$ grau incompleto. A maioria dos pais exercia funções de nível não qualificado ou de qualificação inferior. A renda per capita variava de $\mathrm{R} \$$ 38,34 a $\mathrm{R} \$ 500,00(M d=124,55)$.

3 O projeto de pesquisa foi aprovado pelo Comitê de Ética do HCFMRP - USP 


\section{Local}

O estudo teve a coleta de dados realizada em salas dos ambulatórios da Neonatologia do Hospital das Clínicas da Faculdade de Medicina de Ribeirão Preto.

\section{Material}

Foram utilizados os seguintes materiais: a) Teste das Matrizes Progressivas Coloridas - Escala Especial (Raven), com padronização brasileira de Angellini, Alves, Custódio e Duarte (1988); b) Escala de Inteligência Wechsler para Crianças (WISC), traduzida e adaptada por Poppovic (1964); c) Avaliação Assistida, com Jogo de Perguntas de Busca com Figuras Geométricas (Linhares, 1996; Linhares \& cols. ,1998; Santa Maria \& Linhares, 1999); d) Escala de Comportamento Infantil A2 de Rutter, traduzida e padronizada por Graminha e Coelho (1994); e) Gravador e Fitas cassete.

\section{Procedimento}

\section{Coleta de Dados}

As três primeiras sessões com a criança realizadas por psicóloga treinada em avaliação psicológica tiveram a seguinte ordem de aplicação: Raven, WISC (Escalas Verbal e de Execução completas) e Avaliação Assistida. Esta consiste em situação de resolução de problema, utilizando-se o Jogo das Perguntas de Busca com Figuras Geométricas, com o delineamento de três fases: inicial sem ajuda, fase com assistência e fase de manutenção, de acordo com procedimento descrito por Linhares (1996), e com o gradiente de mediação na fase de assistência conforme proposto por Santa Maria e Linhares (1999). Na quarta sessão a mãe foi solicitada a responder a Escala de Rutter. As sessões tiveram espaçamento semanal.

\section{Análise dos Dados}

Os testes psicométricos de inteligência foram quantificados de acordo com as normas dos mesmos e obtidos o percentil na escala de Raven e o nível intelectual no WISC. Os dados da Avaliação Assistida foram analisados segundo o sistema de categorias, definidas operacionalmente, proposto por Linhares (1996) e Santa Maria e Linhares (1999), quanto aos indicadores de desempenho na tarefa de resolução de problemas: relevância das perguntas de busca de informação e poder de restrição de alternativas (perguntas relevantes, irrelevantes, incorretas ou repetidas) e tipos de tentativas de resolução dos itens (corretas, incorretas e corretas ao acaso). A partir desses indicadores, verificava-se o desempenho potencial na Avaliação Assistida, revelado pela comparação entre o desempenho na fase inicial sem ajuda e as fases de assistência e de manutenção (após a suspensão de assistência), respectivamente. Os sujeitos eram então classificados em termos de perfis de desempenho cognitivo: alto escore (AE), ganhador mantenedor (GM), ganhador dependente da assistência (GDA) e não ganhador (NG), cujas definições operacionais encontram-se descritas em e Linhares e cols. (1998) e Santa Maria e Linhares (1999). Em geral, a criança alto escore, já na fase inicial sem ajuda, apresenta estratégias eficientes de perguntas relevantes de busca e acertos; a ganhadora mantenedora é aquela que melhora ou mantém o desempenho na fase de assistência em relação à fase inicial sem ajuda, assim como mantém o ganho do desempenho na fase de manutenção; a ganhadora dependente da assistência é aquela que melhora ou mantém o desempenho na fase de assistência em relação à fase inicial sem ajuda, mas não mantém o ganho no desempenho na fase de manutenção e a não ganhadora é aquela que não melhora o desempenho na fase de assistência em relação à fase inicial sem ajuda.

Na avaliação da Escala de Rutter foi atribuída a cada item uma pontuação de zero a dois, considerando-se: valor 0 (zero) = ausente, os itens que, na avaliação da mãe, nunca se aplicavam a seu filho; valor $1(\mathrm{um})=$ moderado, os itens que se aplicavam ocasionalmente e valor 2 (dois) = severo, os itens que se aplicavam com certeza. Em seguida, calculava-se a pontuação geral de cada criança, para então, compará-la com a padronização de Graminha e Coelho (1994), que indica o escore maior que 16 como sugestivo da necessidade de atendimento psicológico ou psiquiátrico.Finalmente, foram calculados os coeficientes de correlação de Spearman entre os resultados na prova de Raven (percentil), WISC (QI Geral, QI Verbal e QI de Execução), Avaliação Assistida (perfil de desempenho cognitivo) e Escala de Rutter (escores total e das sub-áreas).

Os dados da avaliação psicométrica e avaliação assistida foram analisados por dois avaliadores independentes especializados em avaliação psicológica e treinados em avaliação assistida. Quando ocorriam dúvidas ou desacordos nos resultados um terceiro avaliador realizava avaliação independente para esclarecimento ou desempate para obtenção da avaliação final.

\section{Resultados}

A Figura 1 apresenta os resultados da avaliação intelectual dos sujeitos através da prova de Raven, em termos de percentil $(n=20)$.

Os resultados obtidos através da aplicação da prova de Raven reunidos na Figura 1 permitem verificar que ocorreu uma amplitude de variação do percentil de 5 a 95 e houve um predomínio de crianças $(65 \%)$ classificadas como definidamente abaixo da média ou deficientes. Destas, a maior parte das crianças apresentaram percentil 25, indicador de inteligência limítrofe e apenas $15 \%$ classificaram-se como deficientes mentais, segundo a padronização brasileira. Foi encontrado ainda que $35 \%$ das crianças estão na média ou acima da média na classificação intelectual da prova de Raven.

A Figura 2 apresenta os resultados da avaliação intelectual das crianças, obtidos através das escalas Geral, Verbal e de Execução do WISC, em termos de classificação do nível 


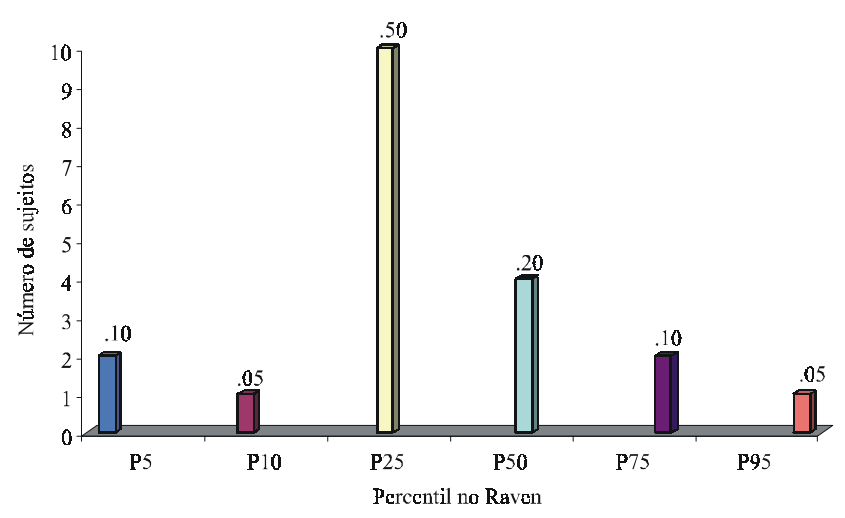

Figura 1. Avaliação intelectual através do Raven - Escla Especial $(n=20)$.

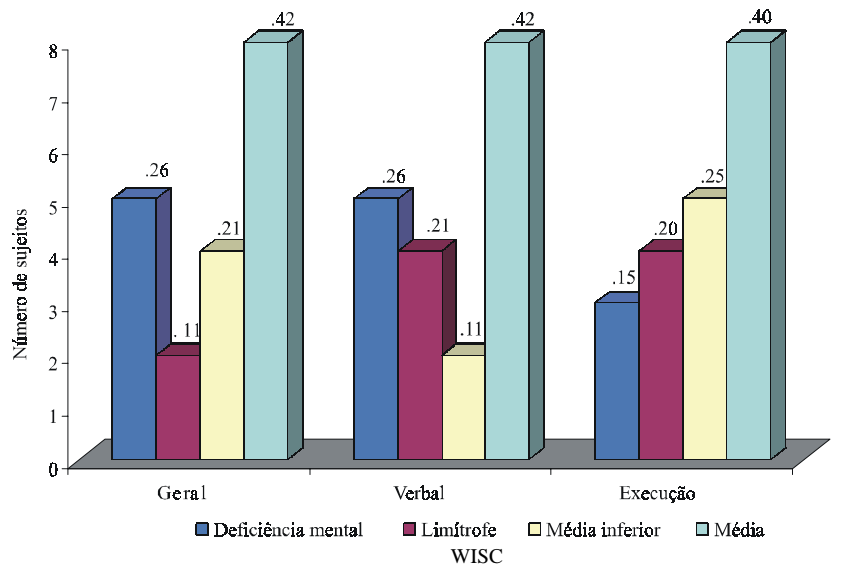

Figura 2. Avaliação Intelectual através do WISC - Classificação nas Escalas Geral $(n=19)$, Verbal $(n=19)$ e de Execução $(n=20)$.

intelectual. Dos 20 sujeitos, um sujeito não conseguiu realizar a Escala Verbal, devido à deficiência auditiva.

A análise dos dados referentes ao teste WISC apresentados na Figura 2 mostra que, com relação à Escala Geral, a maioria das crianças (63\%) apresentou nível intelectual médio e apenas $26 \%$ classificaram-se como deficientes mentais. Na Escala Verbal, permanece a mesma tendência da Escala Geral, sendo 53\% crianças com resultados na média e $26 \%$ com classificação de deficiência mental. Na Escala de Execução, por sua vez, permanece a tendência de crianças na média (65\%). Entretanto, menos crianças classificaram-se como deficientes mentais (15\%) em relação à Escala Verbal. Os dados apresentados a seguir referem-se à avaliação assistida em situação de resolução de problema envolvendo estratégias de perguntas de busca de informação e restrição de alternativas. Dos 20 sujeitos, dois não realizaram esta avaliação, visto que a aplicação de tal procedimento tornou-se inviável para as crianças portadoras de deficiência auditiva, por tratar-se de procedimento que depende fundamentalmente de verbalização para a compreensão e realização da tarefa.

A Figura 3 mostra a distribuição das crianças de acordo com o perfil de desempenho cognitivo obtido através da avaliação assistida.

Levando-se em conta o desempenho de cada criança na avaliação assistida (Jogo de Perguntas de Busca com Figu-

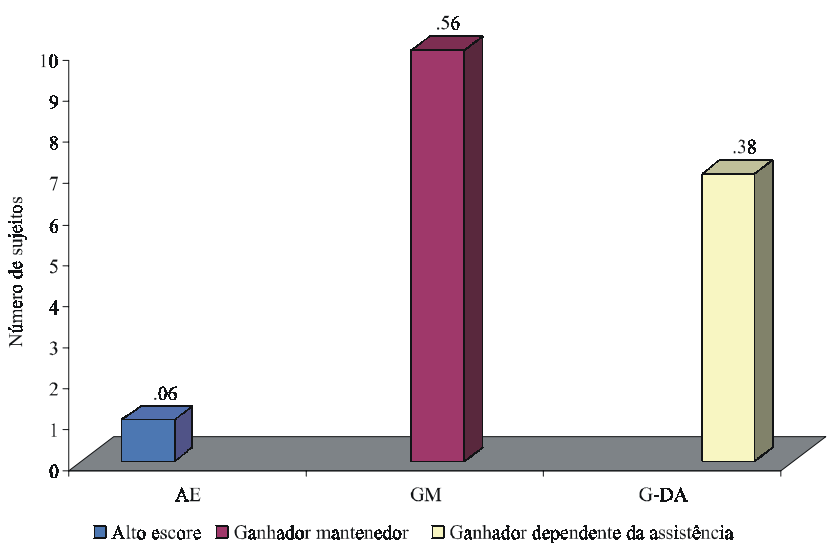

Figura 3. Perfil de Desempenho Cognitivo na Avaliação Assistida $(n=18)$.

ras Geométricas), especificamente o número de perguntas de busca relevantes formuladas e o número de acertos quando comparadas a fase inicial sem ajuda com as fases de assistência e de manutenção, respectivamente, percebe-se que dez crianças (56\%) classificaram-se como ganhadores mantenedores, ou seja, melhoraram seu desempenho na fase de assistência em relação à fase inicial sem ajuda, assim como mantiveram o ganho no desempenho na fase de manutenção. Outras sete crianças (38\%) classificaram-se como $g a$ nhadores dependentes da assistência da examinadora, isto é, melhoraram seu desempenho na fase de assistência em relação à fase inicial sem ajuda mas, em contrapartida não mantiveram o ganho no desempenho na fase manutenção. Apenas uma criança classificou-se como alto escore, isto é, apresentou proporções de perguntas relevantes de busca e de acertos na fase inicial sem ajuda, independente da ajuda da examinadora, conseguindo mantê-las até a fase de manutenção.

Com relação à avaliação do comportamento, segundo a percepção das mães, avaliado através da Escala de Comportamento Infantil A2 de Rutter, verificou-se que $75 \%$ das crianças avaliadas apresentaram escores acima de 16, o que, segundo a padronização da escala realizada com escolares de Ribeirão Preto, este índice constitui-se em indicador de necessidade de atendimento psicológico ou psiquiátrico.

O escore total médio obtido na Escala de Rutter foi de 23, com amplitude de variação de 10 a 37 . Os tipos de comportamentos que mais incidiram $(\geq 70 \%)$, em ordem de maior ocorrência, na amostra estudada foram: agitação (85\%), impaciência (80\%), não permanência nas atividades (75\%), agarramento à mãe $(75 \%)$, dor de cabeça $(75 \%)$, mau humor $(75 \%)$ e preocupação $(70 \%)$

A Tabela 1 mostra os coeficientes de correlação de Spearman referentes aos resultados da avaliação dos sujeitos na prova de Raven (percentil), WISC (QI Geral, QI Verbal e QI de Execução), Avaliação Assistida (perfil de desempenho cognitivo) e Escala de Comportamento Infantil A2 de Rutter (Escore total e escores nas sub-áreas Saúde (S), Hábitos (H) e Comportamento (C).

Os dados da Tabela 1 mostram correlação positiva significativa entre os procedimentos utilizados para a avaliação 
cognitiva, indicando que os sujeitos com os melhores resultados em um procedimento de avaliação mantêm seu desempenho nas demais provas. Pode-se afirmar que as crianças que obtêm os melhores resultados no Raven são as mesmas com os melhores desempenhos no WISC e na avaliação assistida, respectivamente, e vice-versa. Por outro lado, existe uma correlação negativa significativa entre a escala de Rutter (escore total) e as duas provas psicométricas (Raven e WISC), indicando que as crianças com os escores mais elevados na Rutter e, portanto, com indicação de problema de comportamento e com maior necessidade de atendimento psicológico ou psiquiátrico, apresentam desempenho mais rebaixado na avaliação psicométrica de inteligência. Essa correlação foi observada no WISC, especificamente nos escores das escalas Geral e de Execução. Pode ser detectada, com relação à escala Rutter, correlação negativa significativa entre os resultados na área de Hábitos e as provas psicométricas de inteligência (Raven, WISC-G, WISC-V e WISC-E), assim como entre esta área e o perfil de desempenho cognitivo na avaliação assistida. A área de Comportamento da escala Rutter, por sua vez, mostrou correlação negativa significativa com as provas Raven e WISC-E.

\section{Discussão}

A vulnerabilidade da criança nascida pré-termo com muito baixo peso $(\leq 1500 \mathrm{~g})$ constitui-se em alto risco para o desencadeamento de problemas de desenvolvimento, especialmente nas áreas cognitiva e de adaptação psicossocial, em diferentes etapas evolutivas. Considerando-se essa condição, foi desenvolvido o presente estudo, com o objetivo de avaliar aspectos cognitivos e comportamentais desse grupo de risco na média meninice, ou seja, na fase escolar.

Com relação à avaliação cognitiva, verificou-se que os resultados obtidos através dos testes psicométricos Raven e WISC foram coerentes entre si. Considerando-se as provas psicométricas, no Raven, metade das crianças encontram-se classificadas com percentil 25, em uma zona limítrofe à faixa de inteligência média. Na outra metade, a maior parte encontra-se na média ou acima e apenas $15 \%$ classificaramse como deficientes. No WISC, mesmo sem ter padronização brasileira, $63 \%$ das crianças apresentaram um padrão de inteligência na média na Escala Geral e $26 \%$ das crianças classificaram-se como deficientes mentais. A mesma tendência pode ser observada nas escalas Verbal e de Execução.

Comparando-se os resultados nas duas provas, verificouse que as crianças tendem a apresentar os mesmos níveis de desempenho nas duas provas psicométricas, em que se verifica uma correlação positiva entre esses dois procedimentos. Esses resultados corroboram os achados de diferentes autores, que encontraram deficiências cognitivas em diferentes amostras de crianças nascidas prematuras e com baixo peso, assim como crianças com recursos cognitivos, com classificações intelectuais na média ou mesmo acima da média (Bradley \& cols.,1993; Carvalho \& cols., no prelo; Dammann \& cols., 1996; Rieck, Arad \& Netzer., 1996; Smedler \& cols.,1992; Waber \& McCornick,1995). Provavelmente estas últimas, quanto à esfera cognitiva, seriam crianças com sinais de resiliência, que são aquelas que enfrentam positivamente as adversidades na sua trajetória de desenvolvimento, apesar de sua condição de vulnerabilidade (Bordin, 2000; Carvalho, 2000; Rutter, 1987; Werner, 1986).

Apesar de a prematuridade e baixo peso de nascimento, assim como as condições orgânicas adversas decorrentes (apgar baixo, PH abaixo de 7.20, hemorragia intracraniana), constituírem-se em um quadro de "estresse perinatal" (Laucht \& cols, 1997), correlacionando-se com deficiência cognitiva, parece ser necessário cautela no estabelecimento dessa relação.

Nem sempre essa relação de nascimento pré-termo - baixo peso e comprometimento cognitivo é linear, conforme demonstrado por Carvalho (2000) e Carvalho e cols. (no prelo). Kalmár (1996) e Bradley e cols. (1994) mostram a importância do ambiente na promoção do desenvolvimento infantil, demonstrando que a qualidade do ambiente familiar tem mais peso que fatores de risco perinatais em sua capacidade para predizer resultados a longo prazo. Crianças nascidas pré-termo com baixo peso vivendo em condições de pobreza, mas experimentando um ambiente com três ou mais fatores protetores (variedade de estimulação, suporte emocional, responsividade parental e aceitação do comportamento infantil) são mais propensas a mostrar sinais de resiliência. $\mathrm{O}$ ambiente, então, assume um papel importante, na medida em que recursos externos podem ser mobilizados no sentido de promover mediação adequada a essas crianças, dando-lhes condições para a ativação dos recursos

Tabela 1. Coeficientes de correlação de Spearman entre os resultados dos diferentes procedimentos utilizados para a avaliação cognitiva: Raven, WISC (WISC-G, WISC-V e WISC-E), Avaliação Assistida (AVASS) e Escala de Comportamento Infantil A2 de Rutter (Rutter-T, Rutter-S, Rutter-H e Rutter-C)

\begin{tabular}{|c|c|c|c|c|c|c|c|c|c|}
\hline & RAVEN & WI SC- G & WI SC-V & WI SC-E & AVASS & Rutter-T & Rutter-S & Rutter-H & Rutter-C \\
\hline RAVEN & 1.00 & & & & & & & & \\
\hline WISC-G & $0.61 *$ & 1.00 & & & & & & & \\
\hline WISC-V & $0.53 *$ & $0.92 *$ & 1.00 & & & & & & \\
\hline WISC-E & $0.64 *$ & $0.92 *$ & $0.74 *$ & 1.00 & & & & & \\
\hline AVASS & $0.54 *$ & $0.85 *$ & $0.81 *$ & $0.79 *$ & 1.00 & & & & \\
\hline Rutter-T & $-0.74 *$ & $-0.51 *$ & $-0.45 *$ & $-0.52 *$ & $-0.51 *$ & 1.00 & & & \\
\hline Rutter- S & 0 & 0.14 & 0.01 & 0.20 & 0.01 & 0.26 & 1.00 & & \\
\hline Rutter - H & $-0.61 *$ & $-0.73 *$ & $-0.70 *$ & $-0.69 *$ & $-0.70 *$ & $0.81 *$ & 0.09 & 1.00 & \\
\hline Rutter - C & $-0.74 *$ & $-0.44 *$ & -0.33 & $-0.51 *$ & $-0.46 *$ & $0.96 *$ & 0.07 & $0.73 *$ & 1.00 \\
\hline
\end{tabular}

$* p \leq 0.05$ 
que lhes permitirão um funcionamento cognitivo dentro de parâmetros satisfatórios.

$\mathrm{Na}$ amostra estudada, duas crianças (10\%) apresentaram deficiência sensorial auditiva, o que, certamente, influenciou os resultados obtidos pelas mesmas nas provas psicométricas de inteligência. A deficiência auditiva tem sido encontrada por outros pesquisadores em amostras de pré-termo de baixo peso (Carvalho, 2000; Gyler, Dudley, Blinkhorn \& Barnett,1993; Novello \& cols., 1992; O’Brien, Soliday \& McCluskey-Fawcett,1995).

Complementando-se a avaliação tradicional psicométrica da inteligência com a abordagem de avaliação cognitiva assistida, conforme recomendação de Brown e Ferrara (1985), Swanson (1995) e Linhares e cols. (1998), foram obtidas informações quanto aos indicadores de recursos cognitivos potenciais para a aprendizagem na amostra estudada. Na situação de resolução de problemas envolvendo perguntas de busca de informação e restrição de alternativas, em uma modalidade de avaliação com assistência da examinadora, verificouse que mais da metade das crianças são "ganhadoras mantenedoras", isto é, melhoram o seu desempenho na tarefa frente à ajuda mediada da examinadora para resolver a tarefa e mantêm os ganhos após a suspensão da ajuda. Isso indica a presença de recursos potenciais dessas crianças, com necessidade de pouco monitoramento para melhorar o seu desempenho e boa sensibilidade à instrução, conforme encontrado em outros estudos com amostras de crianças com desvantagens, tais como: dificuldade de aprendizagem, deficiência mental ou desvantagem cultural (Brown \& Ferrara,1985; Ferrioli \& Linhares, no prelo; Linhares, 1996; Linhares e cols., 1998; Santa Maria \& Linhares, 1999; Swanson,1995).

Observou-se outro subgrupo menor de crianças que melhoraram o desempenho com a ajuda da examinadora, mas que precisaram de maior quantidade de pistas e tempo de monitoramento para obter sucesso na resolução tarefa. Apenas a otimização da avaliação, através do suporte instrucional temporário oferecido pela examinadora à criança, não foi suficiente para a manutenção da melhora do seu desempenho e de suas estratégias de resolução do problema. Essas crianças sinalizam a necessidade de mediação de aprendizagem intensiva e prolongada em relação às demais, podendo desempenhar-se melhor, desde que efetivamente mediadas em processos de intervenção do tipo educacional ou terapêutica. Cabe salientar que nenhuma criança classificou-se como "não ganhadora", diferentemente dos resultados encontrados em outros estudos com amostras de crianças deficientes mentais e/ou com dificuldade de aprendizagem (Linhares, 1996; Santa Maria \& Linhares, 1999) .

Observou-se neste estudo correlação positiva entre os diferentes resultados na avaliação cognitiva, indicando que: quando havia rebaixamento intelectual, verbal ou não verbal, observava-se a necessidade de maior assistência da examinadora para a criança resolver eficientemente a situação de resolução de problemas. Por outro lado, quando as crianças apresentavam desempenho médio nas avaliações psicométricas de inteligência, apresentavam bom desempenho, com manutenção de ganhos, autonomia e independên- cia da ajuda da examinadora na tarefa de resolução de problemas.

Isso indica que as crianças com rebaixamento intelectual da amostra estudada mostraram-se pouco sensíveis à otimização da avaliação assistida, indicando poucos recursos potenciais e necessidade de maior monitoramento, diferentemente dos sujeitos com dificuldade de aprendizagem com indicação de deficiência cognitiva do estudo de Santa Maria e Linhares (1999), que revelaram um subgrupo que se beneficiava com a assistência e melhorava o seu desempenho apenas com a otimização da situação de avaliação.

Os resultados dos três procedimentos utilizados para a avaliação cognitiva são, portanto, coerentes entre si e demonstram que: a) de maneira geral, as crianças apresentam sinais de funcionamento cognitivo com indicação de capacidades e recursos; b) houve uma tendência a um bom desempenho cognitivo mesmo no WISC, que consiste em um teste sem padronização brasileira; mais da metade das crianças obteve classificação média de desempenho intelectual nas três escalas; c) embora haja uma tendência favorável para o bom funcionamento cognitivo, não deve ser descartada a preocupação com as crianças que apresentam sinais de deficiência, como pode ser observado em uma parcela encontrada na amostra estudada; d) há uma parcela de $50 \%$ de crianças com desempenho limítrofe na escala de Raven em uma "zona de risco", assim como 38\% de crianças que apresentam perfil de desempenho na avaliação assistida que as classifica como "ganhadores dependentes da assistência", denotando a necessidade de monitoramento para obtenção de sucesso no desempenho de tarefa de resolução de problema.

Confirmando os recursos detectados na área cognitiva da amostra estudada quanto à aprendizagem, pode ser observado que em sua maioria esta apresenta bom desempenho escolar, cursando série compatível com a idade cronológica. Apenas duas crianças apresentam história de repetência escolar e outras duas estão em classe especial. Esses resultados demonstram que, no grupo estudado, todas as crianças estão na escola enfrentando o desafio, específico da fase da média meninice, da tarefa evolutiva de "produtividade" ou realização, formulada na teoria psicossocial de Erik Erikson (Erikson, 1971) para crianças na fase escolar.

Com relação ao comportamento, avaliado através da Escala de Rutter, a maioria das mães relatam queixas comportamentais nos filhos, em nível sugestivo de indicação da necessidade de atendimento psicológico ou psiquiátrico. Os problemas apresentados caracterizam o padrão de comportamento da "criança difícil", com sinais de desadaptação psicossocial, envolvendo, de um lado, agitação, impaciência, não permanência nas atividades e por outro lado, oscilação de humor, preocupação e agarramento à mãe. Esse resultados corroboram os achados de Carvalho e cols. (no prelo) e indicam a relevância que deve ser atribuída aos cuidados no manejo da regulação do comportamento e na implementação de práticas educativas adequadas às necessidades de cada criança.

Destaca-se o fato de haver correlação entre as deficiências intelectuais e problemas comportamentais. Sugere, nesse 
sentido, que na amostra estudada existe um subgrupo de crianças com comprometimentos tanto na esfera cognitiva quanto na comportamental, que requer intervenção especializada educacional e terapêutica para mediar o seu desenvolvimento.

\section{Considerações Finais}

As crianças nascidas pré-termo e baixo peso avaliadas neste estudo apresentam dificuldades maiores na área comportamental que na área cognitiva, embora sejam identificadas algumas crianças com um nível intelectual limítrofe ou abaixo da média necessitando de suporte instrucional adicional mediado para ativar recursos potenciais de aprendizagem e desenvolvimento.

Confirmam ser um grupo de risco, com uma parcela que requer intervenção especializada, isto é, crianças que podem ser beneficiadas através de programas efetivos de intervenção educacional ou terapêutica. Liaw e Brooks-Gunn (1993) demonstraram que o desempenho cognitivo de crianças pré-termo e baixo peso está associado à participação precoce em programas de intervenção, os quais, segundo Achenbach (1992), devem voltar-se mais à promoção do desenvolvimento que ao alívio de desconfortos ou à remoção de sintomas.

A condição de vulnerabilidade advinda da prematuridade e do baixo peso não pode ser considerada apenas sob o aspecto de risco ao desenvolvimento mas sim, e acima de tudo, pela identificação e busca de mecanismoss protetores presentes no interjogo entre risco e recurso, conforme proposto por Lewis e cols. (1988) e Linhares, Carvalho, Bordin e Jorge (no prelo). Assim, fica claro que a condição de baixo peso e prematuridade não deve ser considerada de maneira isolada quando se busca a compreensão do impacto no decorrer do desenvolvimento da criança. Considerá-la como fator operante de um sistema bastante complexo, em que diversos aspectos encontram-se inter-relacionados em contexto específico e individual, determinando as formas de interação do indivíduo com o meio, é a maneira mais recomendável.

\section{Referências}

Achenbach, T.M. (1992). Developmental Psychopatology. Em: M. H. Bornstein e M.E. Lamb (Orgs.), Developmental Psychology: an advanced textbook.(pp.629-676).New Jersey: Lawrence Erlbaum Associates.

Angellini, A.L.; Alves, I.C.B. ; Custódio, E.M. \& Duarte, W.F. (1988) Matrizes Progressivas Coloridas de Raven: Escala Especial.. (Padronização brasileira). São Paulo: Casa do Psicólogo.

Antunha, E.L.G. (1994) A avaliação neuropsicológica na infância (0 a 6 anos). Em: V.B. de Oliveira \& N.A. Bossa (Orgs.) Avaliação psicopedagógica da criança de zero a seis anos (pp. 89122). Petrópolis: Vozes.

Beckwith, L. \& Rodning, C. (1991). Intellectual functioning in children born preterm: recent research. Em L. Okagaki \& R.J. Sternberg (Orgs.), Directors of development:influences on the development of the children's thinking (pp. 25-58).Hillsdale, N. J.: Lawrence Erlbaum Associates.
Bernardes, B. (1998). Estudo traça perfil de recém-nascidos. Folha de São Paulo.

Bordin, M.B.M. (2000). Aspectos cognitivos, emocionais e comportamentais na fase escolar de crianças nascidas baixo peso: estudo comparativo entre crianças muito baixo peso $(\leq 1500 \mathrm{~g})$ e crianças baixo peso limítrofe (2000 a 2500g). Dissertação de Mestrado, Universidade de São Paulo, Ribeirão Preto.

Bradley, R.H.; Whiteside, L., Caldwell, B.M., Casey, P.H., Kelleher, K., Pope, S., Swanson, M., Barret, K. \& Cross, D. (1993). Maternal IQ, the home environment, and child IQ in low birthweight, premature children. International Journal of Behavioral Development, 16, 61-74.

Bradley, R.; Whiteside, L.; Mundfrom, D.; Casey, P.H.; Kelleher, K.J. \& Pope, S.K. (1994). Early indications of resilience and their relation to experiences in the home environments of low birthweight, premature children living in poverty. Child Development, 65, 346-360.

Brandt, P.; Magyary, D.; Hammond, M. \& Barnard, K. (1992). Learning and behavioral-emotional problems of children born pre-term at second grade. Journal of Pediatric Psychology, 17, 291-311.

Brazelton, T.B.(1994). Momentos decisivos do desenvolvimento infantil.(J.L. Camargo, Trad.) São Paulo: Martins Fontes. (Trabalho original publicado em 1992).

Brown, A.L. \& Ferrara, R.A. (1985). Diagnosing zone of proximal development. Em: J.V. Wertsch (Org.). Culture, Communication and Cognition: Vygotskian perspectives (pp. 273-305). Cambridge: Cambridge University Press.

Carvalho, A.E.V. (2000). Sobrevivência e qualidade de vida: história de desenvolvimento, aprendizagem e adaptação psicossocial de crianças nascidas pré-termo e com muito baixo peso (< $1500 \mathrm{~g}$ ) em comparação com crianças nascidas a termo. Dissertação de Mestrado, Universidade de São Paulo, Ribeirão Preto.

Carvalho, A.E.V.; Linhares, M.B.M. \& Martinez, F.E. (no prelo). História de desenvolvimento e comportamento de crianças prematuras e de baixo peso $(<1500 \mathrm{~g})$. Psicologia: Reflexão e Crítica.

Dammann, O.; Walther, H.; Allers, B.; Schröder, M.; Drescher, J.; Lutz, D.; Veelken, N. \& Schulte, F.J. (1996). Development of a regional cohort of very low-birth weight children at six years cognitive abilities are associated with neurological disability and social background. Developmental Medicine and Child Neurology, 38 (2), 97-108.

Erikson, E. (1971). Oito idades do homem. Em E.H. Erikson, Infância e sociedade (G. Amado, Trad.). Rio de Janeiro: Zahar (Trabalho original publicado em 1950).

Ferrioli, S.T. \& Linhares, M.B.M. (no prelo). Indicadores de potencial de aprendizagem obtidos através da avaliação cognitiva assistida. Psicologia: Reflexão e Crítica.

Fitzhardinze, P.M. \& Steven, E.M. (1972). The small-for-date infant II. Neurological and intellectual sequelas. Pediatrics, 50 (1), 50-57.

Forslund, M. \& Bjerre, I. (1990). Follow-up of pre - term children: II. Growth and development at four years of age. Early Human Development, 24, 107-118.

Gennaro, S.; Brooten, D.; Roncoli, M. \& Kumar, S.P. (1993). Stress and health outcomes among mothers of low - birth - weight infants. Western Journal of Nursing Research, 15 (1), 97 - 113. 
Graminha, S.S.V. e Coelho, W.F. (1994). Problemas emocionais/ comportamentais em crianças que necessitam ou não de atendimento psicológico ou psiquiátrico. Comunicações Científicas da XXIV Reunião Anual de Psicologia da SBP (p. 263). Ribeirão Preto: SBP.

Gyler, L.; Dudley, M.; Blinkhorn, S. \& Barnett, B. (1993). The relationship between psychosocial factors and developmental outcome for very low and extremely low birthweight infants: a review. Australian and New Zealand Journal of Psychiatry. 27, 62-73.

Kalmár, M. (1996). The course of intellectual development in preterm and fullterm children: an 8-year longitudinal study. International Journal of Behavioral Development, 19, 491-516.

Landry, S.H.; Chapieski, M.L.; Richardson, M.A.; Palmer, J. \& Hall, S. (1990). The social competence of children born prematurely: effects of medical complications and parent behaviours. Child Development, 61, 1605-1616.

Laucht, M.; Esser, G. \& Schmit, M.H. (1997). Developmental outcome of infants born with biological and psychosocial risks. Journal Child Psychology and Psychiatry, 38, 843-853.

Levy-Shiff, R.; Einat, G.; Har-Even, D.; Mogilner, M.; Mogilner, S.; Lerman, M. \& Krikler, R. (1994). Emotional and behavioral adjustment in children born prematurely. Journal of Clinical Child Psychology, 23, 323-333.

Levy-Shiff, R.; Einat, G.; Mogilner, M.B.; Lerman, M. \& Krikler, R. (1994). Biological and environmental correlates of developmental outcome of prematurely born infants in early adolescence. Journal of Pediatric Psychology, 19, 63-78.

Lewis, R.J., Dlugokinski, Caputo, L.M. \& Griffin, R.B. (1988) Children at risk for emotional disorders: risk and dimensions. Clinical Psychology Review, 8, 417-440.

Liaw, F.R. \& Brooks-Gunn, J. (1993). Patterns of low-birth-weight children's cognitive development. Developmental Psychology, 29, 1024-1035.

Linhares, M.B.M. (1996). Avaliação assistida em crianças com queixa de dificuldade de aprendizagem. Temas em Psicologia, 1,17-32.

Linhares, M.B.M.; Santa Maria, M.R.; Escolano, A.C.M. \& Gera, A.A. (1998). Avaliação cognitiva assistida: uma abordagem promissora na avaliação cognitiva de crianças. Temas em Psicologia, 7, 231-254.

Linhares, M.B.M.; Carvalho, A.E.V.; Bordin, M.B.M. \& Jorge, S. M. (no prelo). Suporte psicológico ao desenvolvimento de bebês prematuros com peso de nascimento < 1500g: na UTI neonatal e no seguimento longitudinal. Temas em Psicologia da SBP.

Mutch, L., Leyland, A. \& McGee, A. (1993). Patterns of neuropsychological function in a low birthweight population. Developmental Medicine and Child Neurology, 35, 943-956.

Novello, A.C., Degraw, C. \& Kleinnan, D.V. (1992). Healthy children ready to learn: an essential collaboration between health and education. Public Health Reports, 107 (1), 3-10.

O'Brien, M.; Solyday, E. \& McCluskey-Fawcett, K. (1995). Prematurity and the neonatal intensive care unit. Em M. C. Roberts (Org.), Handbook of pediatric psychology (pp. 463-478). New York, N.Y.: The Guilford Press.

Poppovic, A. (1964). Escala Wechsler de Inteligência para Crianças (WISC) (Tradução e adaptação brasileira). Rio de Janeiro: CEPA
Rieck, M.; Arad, I. \& Netzer, D. (1996). Developmental evaluation of very-low-birthweight infants: longitudinal and cross-sectional studies. International Journal of Behavioral Development, 19, 549-562.

Rutter, M. (1987). Psychosocial resilience and protective mechanisms. American Orthopsychiatric, 57, 316-331.

Sansavini, A.; Rizzardi, M.; Alessandroni, R. \& Giovanelli, G. (1996). The development of italian low and very - low birthweight infants from birth to 5 years: the role of biological and social risks. International Journal of Behavioral Development, 19, 533-547.

Santa Maria, M.R. \& Linhares, M.B.M. (1999). Avaliação cognitiva assistida de crianças com indicações de dificuldades de aprendizagem escolar e deficiência mental leve. Psicologia: Reflexão e Crítica, 12, 395-417.

Smedler, A.C., Faxelius, G., Bremme, K. \& Lagerstrom, M. (1992) Psychological development in children born with very low birthweight after severe intrauterine growth retardation: a 10year follow-up study. Acta Paediatrica, 81, 197-203.

Soares, N.E. \& Fernandes, L.M. (1989). A medida do nível sócioeconômico-cultural. Arquivos Brasileiros de Psicologia, 41 (2), $35-43$.

Sobotková, D.; Dittrichová, J. \& Mandys, F. (1996). Comparison of maternal perceptions of preterm and fullterm infants. Early Development and Parenting, 5 (2), 73-79.

Stjernqvist, K. (1993). The early mother-infant interaction in the highly technological care environment. Acta Pediatrica. 82, 981-982.

Swanson, H.L. (1995). Effects of dynamic testing on the classification of learning disabilities: the predictive and discriminant validity of the Swanson-Cognitive Processing Test (S-CPT). Journal of Psychoeducational Assessment, 13, 204 -229.

Taylor, H.G., Hack, M.; Klein, N. \& Schatschneider, C. (1995). Achievement children with birth weight less than 750 grams with normal cognitive abilities: evidence for specific learning disabilities. Journal of Pediatric Psychology, 20, 703-719.

Tzuriel. D. \& Klein, P.S. (1987) Assessing the young child: children's analogical thinking modifiability. Em C.S. Lidz (Org.), Dynamic assessment - an interactional approach to evaluating learning potential (pp. 268-287). New York: The Guilford Press.

Waber, D.P. \& McCornick, M.C. (1995) Late neuropsychological outcomes in preterm infants of normal IQ: selective vulnerability of the visual system. Journal of Pediatric Psychology, 20, 721-735.

Weiglas-Kuperus, N.; Koot, H.M.; Baerts, W.; Fetter, W.P.F. \& Sauer, P.J.J. (1993). Behaviour problems of very lowbirthweight children. Developmental Medicine and Child Neurology. 35, 406-416.

Werner, E.E. (1986). A longitudinal study of perinatal risk. Em: D.C. Farran \& J. MacCkinney. Risk in intellectual and psychosocial development. N.Y.: Academic Press.

Recebido em 03.07.2000

Primeira decisão editorial em 09.05.2001

Versão final em 28.06.2001

Aceito em 02.07.2001 